\title{
Study on the Application of Markov Random Field in Target Tracking Sun Qing ${ }^{1, a}$, ZHANG Qiliang ${ }^{1, b}$ \\ ${ }^{1}$ The Air-defense and Anti-missile Institute, Air Force Engineering University. Shannxi Xi,an 710051,China

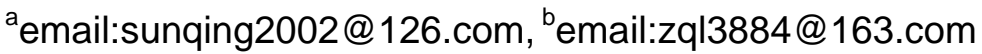

Keywords: Markov Random Field(MRF);Target tracking; Moving target detection.

\begin{abstract}
Being a key link in modern imaging tracking system, the quality of moving target detection in dynamic scene will influence the performance of the whole tracking system immediately. Markov Random Field theory has been successfully used in image target tracking and identification leaning against its complete mathematical theory. In this paper, characteristics of Markov Random Field theory have been discussed and the application in target detection has been analyzed, which supplies some reference to fundamental research and implementation.
\end{abstract}

\section{Introduction}

Markov Random Field, being a kind of probability graph model, is also called Markov net or undirected graph model. In 1974, an English statistician named Julian Besag, led Markov Random Field in image processing field to describe some space correlation characteristics among image elements $^{[1]}$. Nowadays, MRF theory has been widely researched in many fields such as image-recovery, image identification, image segmentation, edge detection, texture analysis, data fusion and so on.

\section{Common Used Methods for Moving Target Detection}

Moving target detection is to judge the existence of moving target in monitoring area and confirm its position, which is generally implemented by video image processing. The detection can be operated in airspace, in time domain, and even in space-time because the video image sequence includes both space information and correlation in time domain. Being an important content in the domain of computer vision, the detecting result of moving target will influence the succeeding work. The interference from ray, weather, shadow of moving target and other factors in actual will make it difficult in moving target detection. Generally speaking, the method of detecting and Subdividing is still immaturity and many unsolved problems exist. Moving target detection can be divided into two classes, one is holding the cam fixed and detecting the visual field targets and most detecting algorithms work on it, the other is based on an unfixed cam and the detection will be complex, and commonly it can be transformed to the first class by compensating the movement of cam. The common used moving target detecting algorithms conclude the difference method of interframe, the deduction method of background, luminous flux method and method based on MRF.

\section{Markov Random Field Model}

Markov Random Field can be described by undirected graph. In Figure 1, according to every position $i$, there will be an implicit state node $x_{i}$ and an observed quantity node $y_{i}$. The edges between nodes express correlation exists between adjacent nodes. In general, the dereferencing of an observed quantity node $y_{i}$ can be acquired by observation directly, however, the state value of an implicit state node $x_{i}$ will be pre-estimated by inference, which is the expected data. The implicit state $x_{i}$ can be a continuous random variable or a discrete one. Here, the word discrete means the state value of every node should come from a volume of discrete candidate states. The problem of solving Markov Random Field model is often treated as the problem of state mark, which means mark every node a state among all the candidate states and make the model acquire the maximum 
posterior probability.

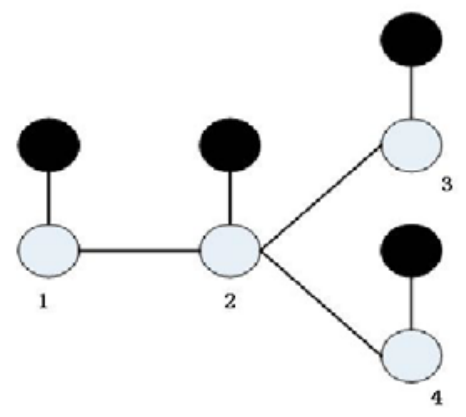

Fig.1 Markov Random Field Model

From Fig. 1 we can see that connection exists between an implicit state node $x_{i}$ and an observed quantity node $y_{i}$ of corresponding position $i$, and the connection can be described by a joint consistency function $\Phi_{i}\left(x_{i}, y_{j}\right)$, and the connection existed between adjacent implicit state nodes corresponding to position $i$ and $j$ can be described by a consistency function $\Psi_{i j}\left(x_{i}, y_{j}\right)$. It can be concluded from the previous analysis that in order to acquire the state value of an implicit state node $x_{i}$ corresponding to position $i$, we need to consider the influences from the observed quantity node $y_{i}$. and the adjacent implicit state node $x_{j}$ integrated to the whole Markov Random Field. And then, a joint distribution function can be get to express the observed field and the implicit state field, showed as Eq.1.

$$
p(\{x\},\{y\})=\frac{1}{Z} \prod_{i \neq j} \psi_{i j}\left(x_{i}, x_{j}\right) \prod_{i} \phi_{i}\left(x_{i}, y_{i}\right)
$$

Here, $Z$ means the normalized constant, and $i \neq j$ means quadrature computation between all the adjacent and mated nodes $i$ and $j$.

Markov Random Field Has Markov property, which makes that the consistency function only exists between mated nodes $i$ and $j$.. However, the nodes in Markov Random Field are interconnection and joint distribution function expresses its global feature appropriately. Thus characteristic is suitable for describe image problems, which means Markov property of mated adjacent pixels can not only react local features of an image, but also effectively describe its global property.

It is difficult to calculate the marginal probability distribution of all nodes according to joint probability distribution function immediately. The algorithm complexity of ergodic the whole solution space will grow exponentially with the increasing of node number, and in general, which will be extraordinary huge in implementation of image processing. Attentions have been paid to the solving methods of Markov Random Field model and two effective ones are solving based on credence propagation and on minimization of free energy.

\section{Applications of Markov Random Field theory in target detection}

Markov Random Field theory has been widely used in many scientific research fields since its proposing from 70's of last century. In 1971, Hamersley and Clifford proved the equivalence between Markov Random Field and Gibbs distribution, which solved the difficulties of calculating Joint probability from conditional probability, modeled MRF theory with an obvious form and extended the application in practice. In 1974, Besag put forword a future joint distribution form of Markov Random Field and which supplied mathematic model for image processing in Bayes system.

The association of MRF, contingency tables and graphs has been further discussed with the profound research. Some scholars have proposed that the pixel expression of an image perhaps was not optimal as to the simulation of dependence relationship in true picture. According with the general features of MRF, and MRF model can be used to simulate an image grain on condition of 
finding a constant group, while the method is limited in grain image.

In 1984, S.German and D.German published an important issue about random field. They approved and applied Hammersley-CHfford theorem in establishing the interconnection between local characteristic and global one of an image, and with which, the prior probability distribution and posterior probability distribution of bivariate image system were expressed and determined by potential function. Being a great progress in the implementation of MRF theory, the research work has expanded the application in image analysis. From then on, MRF has been used in many fields, changed from single MRF model to Double one, and finally been developed to layering MRF model, and with the causality between layers, non-iterative algorithm is deduced, which processes lower complexity and higher accuracy than the primary non-causality MRF model.

Great progress has been achieved in China. In 2003, Wang Xili and her team members put forward a new layering MRF model and algorithm and improved to a half tree model. The model has the characteristics of high speed and short error classification like the convential MRF model, and it even simplify the problems and solving mechanism. In 2004, Cheng Bing and others combined MRF model with FRAME and proposed a no-supervision division method of multi-grain. Compared with traditional MRF model, FRAME model can access value in a wider neighborhood system, and process model to more complex image style.

Markov Random Field theory has been applied to many fields such as computer vision and image processing, and the application including image-recovery and rebuilding, movement estimation, texture analysis, edge detection, image segmentation, target identification, attitude estimation and so on. The principle is using conditional probability, which depends on the relative information about the neighborhood of an image point and is independent on its position, is to describe image data distribution. According to the conformance between MRF and Gibbs distribution to determine the conditional probability of MRF by potential function and make it process the global consistency. Combined with Bayes criterion, MRF field theory can transform problems into the combination optimization of solving minimum energy function. There are three common solving schemes, Simulated Annealing algorithm, Maximiser of Posterior Marginals (MPM) algorithm and Iterated Conditional Models(ICM). Compared the three algorithms, ICM processes the highest computational efficiency, but it will be converged to the locally optimal solution net. MAP algorithm is the most used criterion in MRF modeling, and combined with MRF model, related patterns between image and pixel can be expressed clearly and visualized by using diagram.

Moving target detection in dynamic scene is a key link in modern imaging tracking, and the detection quality will influence the performance of the whole tracking system. The detecting method based on MRF uses the local coherent statistic characteristics of an image to describe correctly the important dependence between the type that every pixel belongs to and the ambient pixels. Scholars home and abroad have done further research on the application of image segmentation based on MRF detecting method and achieved vital achievements. In reference [3], a moving target detecting method based on regional maximum posterior probability is proposed and reference [4] gives out a moving target detecting method which combined newly established MRF model of double-scale neighborhood with the dividing algorithm improved from non-linear transformation. In reference [5], color cluster of original image is used as a priori knowledge to redefine energy function to achieve moving target detection and in reference [6], EM/E-MRF adaptive model is applied in target detection and good result is achieved.

\section{Conclusion}

Markov Random Field theory has been successfully applied in image target tracking and identification and has extensive implementation prospect in multiple-dimensioned image statistics and modeling for its complete mathematical theory and strong containment. Based on the characteristics of Markov Random Field theory, we do some analysis work on the application in moving target detection. However, the detection faces many difficulties such as complex cause to noisy images and the diversification of environment. Further research about theory and technique 
on the application of Markov Random Field theory in moving target detection will be improved and completed.

\section{References}

[1] SUN Liye,WU Kanzhi. Tree-Structured MRF Based Image Segmentation Combined with Advanced Means Shift Mode Detection[C].2011 Sixth International Conference on Image and Graphics, 2011.

[2] DING Ying, LI Wenhui, FAN Jingtao, YANG Huamin. Medical image registration algorithm based on the SAM of multi-scale Harris corner[J].Journal of Image and Graphics,2010.

[3] BAO Hongqiang, ZHANG Zhaoyang. Algorithm of video object segmentation based on region Gibbs potential function[J].Journal of Communications,2005,6(26).

[4] YANG Wenming, LIU Jilin, WANG Qicong. Automatic ex-traction of video object plane by integrating spatio-temporal information[J]. Journal of Computer-aided Design \& Computer Graphics, $2006,6(18)$.

[5] HUANG Xianwu, ZHU Li, ZHONG Xingrong, WANG Jia-jun. A novel moving object segmentation technology based on spatiotemporal Markov random field[J]. Journal of Electronics and Information Technology, 2006, 28(4).

[6] STOLKIN R, GREIG A, HODGETTS M, GILBY J. An EM/E-MRF algorithm for adaptive model based tracking in extremely poor visibility[J]. Image and Vision Computing, 2008.

[7] QIU Chenguang. Moving target detection and tracking in dynamic image sequences[D]. Harbin:Harbin Engineering University, 2010:30-43. 